The Bulletin of Symbolic Logic

Volume 1, Number 2, June 1995

\title{
IN MEMORIAM: CHRISTOPHER JOHN ASH
}

Christopher John Ash died in February, 1995, at the age of 50. Ash was based at Monash University in Australia, throughout his career, having gone there from Oxford with John Crossley, his thesis advisor. Ash held visiting positions in the United States, at the University of Connecticut, the University of Wisconsin, and Notre Dame. He was a Fellow of the Australian Academy of Science.

Within logic, Ash worked on a variety of problems, but over the past 15 years, he focused mainly on a specific program in recursive structure theory. This program, which grew out of joint work with Nerode, involved finding syntactical conditions which correspond to bounds on recursive complexity that persist when a given structure is copied. For a recursive structure $\mathfrak{A}$ and a recursive relation $R$ on $\mathfrak{A}$, Ash and Nerode gave conditions guaranteeing that in all recursive copies of $\mathfrak{A}$, the image of $\mathrm{R}$ is r.e. Ash asked Ewan Barker (then working on a master's degree) to generalize this, replacing r.e., by $\Sigma_{\alpha}^{0}$, for an arbitrary recursive ordinal $\alpha$. Producing a recursive copy of $\mathfrak{A}$ in which the image of $R$ is not r.e., involved a finite injury priority construction. Producing a recursive copy in which the image of $R$ is not $\Sigma_{\alpha}^{0}$ required a much more complicated construction. Ash formulated in an abstract way the object of a nested priority construction, and he proved a general "metatheorem," listing conditions which guarantee the success of the construction. Barker used Ash's metatheorem. There have been many other applications, and there are variants of the original metatheorem with further applications.

In addition to his work in logic, Ash produced several deep results in algebra. At Monash, colleagues interested in semigroups discussed their work with him, and stated for him some of the important open problems in their field. Ash was able to solve some of these problems. In particular, he proved a long-standing conjecture of John Rhodes.

Chris and I collaborated on 10 papers already published, with others in progress. He was full of ideas, an excellent correspondent, and fun to talk with. I enjoyed every minute I had with him.

JULIA F. KNIGHT 